\title{
ON NEW CLASSES OF ANALYTIC FUNCTIONS IMPOSED VIA THE FRACTIONAL ENTROPY INTEGRAL OPERATOR
}

\author{
Rabha W. Ibrahim
}

\begin{abstract}
In this paper, we aim to introduce some geometric properties of analytic functions by utilizing the concept of fractional entropy in a complex domain. We extend the fractional entropy, type Tsallis entropy in the complex z-plane, by using some analytic functions. Established by this diffusion, we state specific new classes of analytic functions (type Schwarz function). Other geometric properties are validated in the sequel. Our development is completed by the Euler form Lemma and Jack Lemma.

Keywords: Fractional calculus; fractional entropy; analytic function; subordination and superordination
\end{abstract}

\section{Introduction}

The theory of operators' apprehensions with the impress of different classes of functions on function spaces, which are given by differential operators and integral operators. The operators may be neglected hypothetically by their categories, such as closed, compact or bounded operators. These information modeled two kinds of operators: linear operators and nonlinear operators. The data itself respite on the topological or geometrical properties of the spaces of functions.

A assembly of exciting physical activities methods to entropic procedures that are accompanying corporate than the ordinary entropy. In 1988, Tsallis offered an original kind of fractional entropy. The Tsallis Entropy has been active close to the Principle of maximum entropy to develop the Tsallis distribution. This entropy has been affianced in many areas such as thermodynamics, statistical mechanics, chaos and information theory. For continuous probability distributions, the entropy is determined by the formal:

$$
\mathcal{T}_{\beta}[v]=\frac{1}{\beta-1}\left(1-\int(v(x))^{\beta} d x\right), \quad \nu \neq 1,
$$

Received June 21, 2016; accepted August 06, 2016

2010 Mathematics Subject Classification. Primary 30C45; Secondary 30C55 
or in the functional form

$$
\mathcal{T}_{\beta}[v](x)=\frac{1}{\beta-1}\left(1-\int_{0}^{x}(v(u))^{\beta} d u\right),
$$

where $v(x)$ is a probability density function.

Here, we generalize this definition into the unit disk $U:=\{z:|z|<1\}$ by using the analytic function $\eta(z), z \in U$ (type Schwarz function $|v(z)|<|z|$,) where it is normalized by $v(0)=0, v^{\prime}(0)=1$. The class of all normalized functions is denoted by $\mathcal{A}_{v}$. For integrals of the second type defined by (see [1]-[2]):

$$
\mathcal{J}_{\beta}[v](z)=\int_{0}^{z}\left(v^{\prime}(t)\right)^{\beta} d t \quad \beta \neq 1, z \in U
$$

we impose the complex Tsallis entropy by

$$
\mathcal{T}_{\beta}[v](z)=\frac{1-\mathcal{J}_{\beta}[v](z)}{\beta-1}, \quad \beta \neq 1, z \in U .
$$

The objective of this paper, is to combine with the differential entropy, because this situation allows us to realize several geometric properties and information concerning the classes of analytic functions. Acceptance facts about the properties of a function from properties of its derivatives permits a large measure in various subjects of mathematical analysis. There are two measures of these concerns characterizations and bound to the function.

The function $v$ has enough information in each geometric classes; such as the starlikeness

$$
\operatorname{Re}\left(z v^{\prime}(z) / v(z)\right)>0
$$

and convexity

$$
\operatorname{Re}\left(1+z v^{\prime \prime}(z) / v^{\prime}(z)\right)>0,
$$

and bounded turning $(\mathcal{B}), \operatorname{Re}\left(f^{\prime}(z)\right)>0$, if the complex entropy satisfies

$$
\operatorname{Re}\left(\mathcal{T}_{\beta}[v](z)\right)>0
$$

which is equivalent to

$$
\operatorname{Re}\left(1-\mathcal{J}_{\beta}[v](z)\right)>0, \quad \beta>1,
$$

where

$$
p(z):=1-\mathcal{J}_{\beta}[v](z), \quad z \in U .
$$

The set of information for each geometric class can be realized by the conclusion

$$
\mathcal{I}_{v}^{\beta}=\left\{v \in A_{v}: 0<\operatorname{Re}\left(\mathcal{T}_{\beta}[v](z)\right)<\frac{\beta}{\beta-1}, z \in U, \beta \neq 1\right\} .
$$


Also, we define a new operator $Q: \mathcal{A} \rightarrow \mathcal{A}$ as follows:

$$
\begin{aligned}
Q_{\beta}[v](z) & :=\frac{(\beta-1)}{A_{2}} \mathcal{T}_{\beta}[v](z) \\
& =\left[1-\frac{\mathcal{J}_{\beta}[v](z)}{z}\right] / A_{2}, \quad \beta \neq 1, A_{2} \neq 0, \\
& =z+\nu_{2} z^{2}+\ldots,
\end{aligned}
$$

where $A_{2}$ is the generalized coefficient of $a_{2}$ of the function

$$
v(z)=z+\sum_{n=2}^{\infty} a_{n} z^{n} .
$$

We call $Q_{\beta}[v](z)$ is the entropy operator generated by he integral operator $\mathcal{J}_{\beta}[v](z)$. We study the geometric properties of this operator in the open unit disk.

Our procedure is founded by the Euler type Lemma as well as Jack Lemma correspondingly (see [4]; P39)

Lemma 1.1. If $p \in H[a, n]$ (the set of analytic functions), then

$$
\operatorname{Re}\left(p(z)+z p^{\prime}(z)+z^{2} p^{\prime \prime}(z)\right)>0 \Longrightarrow \operatorname{Re}(p(z))>0 .
$$

Lemma 1.2. [3] Let $\phi(z)$ be analytic in $U$ with $\phi(0)=0$. Then if $|\phi(z)|$ approaches its maximization when $|z|=r$ at a point $z_{0} \in U$, then $z_{0} \phi^{\prime}\left(z_{0}\right)=\kappa \phi\left(z_{0}\right)$, where $\kappa \geq 1$ is a real number.

Furthermore, we requisite the subordination impression in the consequence, which is demarcated as follows: Let $\omega(z)$ and $w(z)$ be two analytic functions in $U$. Then $\omega(z)$ is said to be subordinate to $w(z)$ if there exists an analytic function $\psi(z)$ in $\mathrm{U}$ achieving $\psi(0)=0,|\psi(z)|<1(z \in U)$ and $\omega(z)=w(\psi(z))$. This subordination is noted by

$$
\omega(z) \prec w(z), \quad z \in U .
$$

\section{The main outcomes}

Our aim is to achieve the property of the set $\mathcal{I}_{v}^{\beta}$.

Theorem 2.1. Let $v \in \mathcal{A}_{v}, \beta \geq 2$, and $3<\sigma<5$. If $\eta$ satisfies

$$
0<\operatorname{Re}\left(\frac{z v^{\prime \prime}(z)}{v^{\prime}(z)}+\frac{2}{\left(\frac{z v^{\prime}(z)}{v(z)}\right)}\right)<\frac{5-\sigma}{2}, \quad \operatorname{Re}(z) \neq 0,
$$

then $v \in \mathcal{I}_{v}^{\beta}$. Moreover, $v$ is starlike in the open unit disk. 
Proof. First, we show that $\operatorname{Re}\left(\mathcal{T}_{\beta}[v](z)\right)<\frac{\beta}{\beta-1}$. Define a function $\psi$ as follows:

$$
\psi(z)=\mathcal{T}_{\beta}[v](z)-\frac{1}{\beta-1}, \beta \neq 1
$$

such that

$$
\psi^{\prime}(z)=\left(\mathcal{T}_{\beta}[v](z)\right)^{\prime}
$$

satisfying the equality

$$
\frac{z v^{\prime}(z)}{v(z)}=\frac{\sigma(1-\psi(z))}{\sigma-\psi(z)}, \quad \psi(z) \neq \sigma .
$$

Since $v$ is analytic in the open disk $U$ then $\psi$ is analytic. In addition, $\psi(0)=0$, we need only to show that $|\psi(z)|<1$. A computation leads to

$$
\begin{gathered}
\operatorname{Re}\left(\frac{z v^{\prime \prime}(z)}{v^{\prime}(z)}+\frac{2}{z} \frac{v(z)}{v^{\prime}(z)}\right)=\operatorname{Re}\left(\frac{\sigma(1-\psi(z))}{\sigma-\psi(z)}-\frac{z \psi^{\prime}(z)}{1-\psi(z)}+\frac{z \psi^{\prime}(z)}{\sigma-\psi(z)}+\frac{\sigma(1+\psi(z))-2 \psi(z)}{\sigma(1-\psi(z))}\right) \\
=\operatorname{Re}\left(\frac{\sigma\left(1-\left[\mathcal{T}_{\beta}[v](z)-\frac{1}{\beta-1}\right]\right)}{\sigma-\left[\mathcal{T}_{\beta}[v](z)-\frac{1}{\beta-1}\right]}-\frac{z\left(\mathcal{T}_{\beta}[v](z)\right)^{\prime}}{1-\left[\mathcal{T}_{\beta}[v](z)-\frac{1}{\beta-1}\right]}+\frac{z\left(\mathcal{T}_{\beta}[v](z)\right)^{\prime}}{\sigma-\left[\mathcal{T}_{\beta}[v](z)-\frac{1}{\beta-1}\right]}\right. \\
\left.\quad+\frac{\sigma\left(1+\mathcal{T}_{\beta}[v](z)-\frac{1}{\beta-1}\right)-2\left[\mathcal{T}_{\beta}[v](z)-\frac{1}{\beta-1}\right]}{\sigma\left(1-\left[\mathcal{T}_{\beta}[v](z)-\frac{1}{\beta-1}\right]\right)}\right) \\
<\frac{5-\sigma}{2}
\end{gathered}
$$

By employing Lemma 1.2, we conclude that there exists a complex number $z_{0} \in U$ such that $\psi\left(z_{0}\right)=e^{i \theta}$ and $z_{0} \psi^{\prime}\left(z_{0}\right)=\kappa \psi\left(z_{0}\right)=\kappa e^{i \theta}, \kappa \geq 1$. Therefore, we obtain

$$
\begin{aligned}
& \operatorname{Re}\left(\frac{z_{0} v^{\prime \prime}\left(z_{0}\right)}{v^{\prime}\left(z_{0}\right)}+\frac{2}{z_{0}} \frac{v\left(z_{0}\right)}{v^{\prime}\left(z_{0}\right)}\right)=\operatorname{Re}\left(\frac{\sigma\left(1-\left[\mathcal{T}_{\beta}[v]\left(z_{0}\right)-\frac{1}{\beta-1}\right]\right)}{\sigma-\left[\mathcal{T}_{\beta}[v]\left(z_{0}\right)-\frac{1}{\beta-1}\right]}-\frac{z_{0}\left(\mathcal{T}_{\beta}[v]\left(z_{0}\right)\right)^{\prime}}{1-\left[\mathcal{T}_{\beta}[v]\left(z_{0}\right)-\frac{1}{\beta-1}\right]}\right. \\
& \left.\quad+\frac{z_{0}\left(\mathcal{T}_{\beta}[v]\left(z_{0}\right)\right)^{\prime}}{\sigma-\left[\mathcal{T}_{\beta}[v]\left(z_{0}\right)-\frac{1}{\beta-1}\right]}+\frac{\sigma\left(1+\mathcal{T}_{\beta}[v]\left(z_{0}\right)-\frac{1}{\beta-1}\right)-2\left[\mathcal{T}_{\beta}[v]\left(z_{0}\right)-\frac{1}{\beta-1}\right]}{\sigma\left(1-\left[\mathcal{T}_{\beta}[v]\left(z_{0}\right)-\frac{1}{\beta-1}\right]\right)}\right) \\
& =\frac{\sigma\left(1-e^{i \theta}\right)}{\sigma-e^{i \theta}}-\frac{\kappa e^{i \theta}}{1-e^{i \theta}}+\frac{\kappa e^{i \theta}}{\sigma-e^{i \theta}}+\frac{\sigma\left(1+e^{i \theta}\right)-2 e^{i \theta}}{\sigma\left(1-e^{i \theta}\right)} \\
& =\frac{\sigma\left(1-e^{i \theta}\right)}{\sigma-e^{i \theta}}-\frac{\sigma \kappa e^{i \theta}}{\sigma\left(1-e^{i \theta}\right)}+\frac{\kappa e^{i \theta}}{\sigma-e^{i \theta}}+\frac{\sigma\left(1+e^{i \theta}\right)-2 e^{i \theta}}{\sigma\left(1-e^{i \theta}\right)} \\
& =\frac{\sigma+(\kappa-\sigma) e^{i \theta}}{\sigma-e^{i \theta}}+\frac{\sigma\left(1+e^{i \theta}-\kappa e^{i \theta}\right)-2 e^{i \theta}}{\sigma\left(1-e^{i \theta}\right)} .
\end{aligned}
$$


Since

$$
\operatorname{Re}\left(\frac{1}{\sigma\left(1-e^{i \theta}\right)}\right)=\frac{1}{2 \sigma}, \quad \operatorname{Re}\left(\frac{1}{\sigma-e^{i \theta}}\right)=\frac{1}{2 \sigma}+\frac{\sigma^{2}-1}{2 \sigma\left(1+\sigma^{2}-2 \sigma \cos (\theta)\right)}
$$

then, we obtain

$$
\operatorname{Re}\left(\frac{z_{0} v^{\prime \prime}\left(z_{0}\right)}{v^{\prime}\left(z_{0}\right)}+\frac{2}{z_{0}} \frac{v\left(z_{0}\right)}{v^{\prime}\left(z_{0}\right)}\right)=\frac{1+\sigma}{2}+\frac{\sigma-1}{\sigma}+\frac{\left(\sigma^{2}-1\right)(2-\sigma+\kappa)}{2\left(1+\sigma^{2}-2 \sigma \cos \theta\right)} .
$$

For $\sigma \in(3,5)$ and $\kappa=1$, we have

$$
\begin{aligned}
\operatorname{Re}\left(\frac{z_{0} v^{\prime \prime}\left(z_{0}\right)}{v^{\prime}\left(z_{0}\right)}+\frac{2}{z_{0}} \frac{v\left(z_{0}\right)}{v^{\prime}\left(z_{0}\right)}\right) & \geq \frac{1+\sigma}{2}+\frac{\sigma-1}{\sigma}+\frac{(\sigma+1)(3-\sigma)}{2(\sigma-1)} \\
& \geq \frac{1+\sigma}{2}+\frac{1-\sigma}{2}+\frac{3-\sigma}{2} \\
& =\frac{5-\sigma}{2},
\end{aligned}
$$

which contradicts the assumption of the theorem. Therefore, $|\psi(z)|<1$, consequently $\operatorname{Re}\left(\mathcal{T}_{\beta}[v](z)\right)<\frac{\beta}{\beta-1}$. Clearly, we have

$$
\begin{aligned}
\psi(z) & =\frac{\sigma\left(\frac{z v^{\prime}(z)}{v(z)}-1\right)}{\frac{z v^{\prime}(z)}{v(z)}-\sigma}, \quad z \in U \\
& =\frac{\sigma\left(1-\frac{z v^{\prime}(z)}{v(z)}\right)}{\sigma-\frac{z v^{\prime}(z)}{v(z)}} .
\end{aligned}
$$

Hence, we have

$$
\frac{z v^{\prime}(z)}{v(z)} \prec \frac{\sigma(1-z)}{\sigma-z}
$$

This implies that $v$ is starlike in $U$. Now, we proceed to show that $\operatorname{Re}\left(\mathcal{T}_{\beta}[v](z)\right)>0$. Since $v$ is starlike, then we introduce a function $p(z)$ such that

$$
p(z)=\frac{v(z)}{z} \Rightarrow z p^{\prime}(z)+p(z)=v^{\prime}(z)
$$

Thus, we obtain

$$
\operatorname{Re}\left(z v^{\prime \prime}(z)+2 \frac{v(z)}{z}-v^{\prime}(z)\right)=\operatorname{Re}\left(p(z)+z p^{\prime}(z)+z^{2} p^{\prime \prime}(z)\right) .
$$

But

$$
\operatorname{Re}\left(z v^{\prime \prime}(z)+2 \frac{v(z)}{z}-v^{\prime}(z)\right)>0 \Leftrightarrow \operatorname{Re}\left(\frac{z v^{\prime \prime}(z)}{v^{\prime}(z)}+\frac{2}{\left(\frac{z v^{\prime}(z)}{v(z)}\right)}\right)>0
$$

By applying Lemma 1.1, we attain $\operatorname{Re}(p(z))>0$ and this leads to $\operatorname{Re}\left(\mathcal{T}_{\beta}[v](z)\right)>0$. This completes the proof. 
A direct application of Theorem 2.1, we have the following result:

Corollary 2.1. Let the hypotheses of Theorem 2.1 hold. Then $\int_{0}^{z} \frac{v(t)}{t} d t$ is convex in the open unit disk.

Theorem 2.2. Consider the entropy operator (1). If for $1<\beta<2$, such that

$$
\operatorname{Re}\left\{\frac{z Q_{\beta}[v]^{\prime \prime}(z)}{Q_{\beta}[v]^{\prime}(z)}\right\}>\frac{\beta}{2},
$$

then $Q_{\beta}[v](z) \in \mathcal{B}$.

Proof. Define a function $\psi(z), z \in U$ as follows:

$$
Q_{\beta}[v]^{\prime}(z)=(1-\psi(z))^{\beta}, \quad z \in U,
$$

where, $\psi(z)$ is analytic with $\psi(0)=0$. We need only to show that $|\psi(z)|<1$. From the definition of $\psi$, we have

$$
\frac{z Q_{\beta}[v]^{\prime \prime}(z)}{Q_{\beta}[v]^{\prime}(z)}=\beta \frac{-z \psi^{\prime}(z)}{1-\beta(z)} .
$$

Hence, we obtain

$$
\begin{aligned}
\operatorname{Re}\left\{\frac{z Q_{\beta}[v]^{\prime \prime}(z)}{Q_{\beta}[v]^{\prime}(z)}\right\} & =\beta \operatorname{Re}\left\{\frac{-z \psi^{\prime}(z)}{1-\psi(z)}\right\} \\
& >\frac{\beta}{2}, \quad \beta \in(1,2) .
\end{aligned}
$$

In view of Lemma 1.2 , there exists a complex number $z_{0} \in U$ such that $\psi\left(z_{0}\right)=e^{i \theta}$ and

$$
z_{0} \psi^{\prime}\left(z_{0}\right)=\kappa \psi\left(z_{0}\right)=\kappa e^{i \theta}, \kappa \geq 1
$$

Therefore, we attain

$$
\begin{aligned}
\operatorname{Re}\left\{\frac{z Q_{\beta}[v]^{\prime \prime}\left(z_{0}\right)}{Q_{\beta}[v]^{\prime}\left(z_{0}\right)}\right\} & =\beta \operatorname{Re}\left\{\frac{-\kappa \psi\left(z_{0}\right)}{1-\psi\left(z_{0}\right)}\right\} \\
& =\beta \operatorname{Re}\left\{\frac{-\kappa e^{i \theta}}{1-e^{i \theta}}\right\} \\
& \leq \frac{\beta}{2}, \quad \kappa=1
\end{aligned}
$$

which contradicts the assumption of the theorem. Hence, there is no $z_{0} \in U$ with $\left|\psi\left(z_{0}\right)\right|=1$, which yields that $|\psi(z)|<1$. Moreover, we have

$$
Q_{\beta}[v]^{\prime}(z) \prec(1-z)^{\beta},
$$

which means that $\operatorname{Re}\left[Q_{\beta}[v]^{\prime}(z)\right]>0$, equivalently, $Q_{\beta}[v]^{\prime}(z) \in \mathcal{B}$. This completes the proof. 
Theorem 2.3. Consider the entropy operator (1.1). If for $0 \leq \beta<1$, such that

$$
\operatorname{Re}\left\{\frac{z Q_{\beta}[v]^{\prime \prime}(z)}{Q_{\beta}[v]^{\prime}(z)}\right\}>\beta
$$

then $Q_{\beta}[v](z) \in \mathcal{S}^{*}$.

Proof. Define a function $\vartheta(z), z \in U$ as follows:

$$
\frac{z Q_{\beta}[v]^{\prime}(z)}{Q_{\beta}[v](z)}=\left(\frac{1+\vartheta(z)}{1-\vartheta(z)}\right)^{\beta}, \quad z \in U,
$$

where, $\vartheta(z)$ is analytic with $\vartheta(0)=0$. We need only to show that $|\vartheta(z)|<1$. From the definition of $\vartheta$, we have

$$
1+\frac{z Q_{\beta}[v]^{\prime \prime}(z)}{Q_{\beta}[v]^{\prime}(z)}=\left(\frac{1+\vartheta(z)}{1-\vartheta(z)}\right)^{\beta}+2 \beta\left[\frac{z \vartheta^{\prime}(z)}{1-\vartheta^{2}(z)}\right]
$$

Hence, we obtain

$$
\begin{aligned}
1+\operatorname{Re}\left\{\frac{z Q_{\beta}[v]^{\prime \prime}(z)}{Q_{\beta}[v]^{\prime}(z)}\right\} & =\operatorname{Re}\left\{\left(\frac{1+\vartheta(z)}{1-\vartheta(z)}\right)^{\beta}+2 \beta\left[\frac{z \vartheta^{\prime}(z)}{1-\vartheta^{2}(z)}\right]\right\} \\
& >\beta, \quad \beta \in[0,1) .
\end{aligned}
$$

In view of Lemma 1.2 , there exists a complex number $z_{0} \in U$ such that $\vartheta\left(z_{0}\right)=$ $e^{i \theta}$ and

$$
z_{0} \vartheta^{\prime}\left(z_{0}\right)=\kappa \vartheta\left(z_{0}\right)=\kappa e^{i \theta}, \kappa \geq 1 .
$$

Therefore, we arrive at

$$
\begin{aligned}
1+\operatorname{Re}\left\{\frac{\left.z Q_{\beta}[v]\right]^{\prime \prime}\left(z_{0}\right)}{Q_{\beta}[v]^{\prime}\left(z_{0}\right)}\right\} & =2 \beta \operatorname{Re}\left\{\frac{z_{0} \vartheta^{\prime}\left(z_{0}\right)}{1-\vartheta^{2}\left(z_{0}\right)}\right\}+\operatorname{Re}\left\{\left(\frac{1+\vartheta\left(z_{0}\right)}{1-\vartheta\left(z_{0}\right)}\right)^{\beta}\right\} \\
& =2 \beta \operatorname{Re}\left\{\frac{\kappa \vartheta\left(z_{0}\right)}{1-\vartheta^{2}\left(z_{0}\right)}\right\}+\operatorname{Re}\left\{\left(\frac{1+\vartheta\left(z_{0}\right)}{1-\vartheta\left(z_{0}\right)}\right)^{\beta}\right\} \\
& =2 \beta \operatorname{Re}\left\{\frac{\kappa e^{i \theta}}{1-e^{2 i \theta}}\right\}+\operatorname{Re}\left\{\left(\frac{1+e^{i \theta}}{1-e^{i \theta}}\right)^{\beta}\right\} \\
& \leq \beta+1, \quad \kappa=1 .
\end{aligned}
$$

This implies that

$$
\operatorname{Re}\left\{\frac{z Q_{\beta}[v]^{\prime \prime}\left(z_{0}\right)}{Q_{\beta}[v]^{\prime}\left(z_{0}\right)}\right\} \leq \beta,
$$

which contradicts the assumption of the theorem. Hence, there is no $z_{0} \in U$ with $\left|\vartheta\left(z_{0}\right)\right|=1$, which yields that $|\vartheta(z)|<1$. Moreover, we have

$$
\frac{z Q_{\beta}[v]^{\prime}(z)}{Q_{\beta}[v](z)} \prec\left(\frac{1+z}{1-z}\right)^{\beta},
$$

which means that $\left.Q_{\beta}[v](z)\right] \in \mathcal{S}^{*}$. 
Theorem 2.4. Consider the entropy operator (1.1). If for $1<\beta<2$, such that

$$
\operatorname{Re}\left\{\frac{z Q_{\beta}[v]^{\prime}(z)}{Q_{\beta}[v](z)}\right\}>\frac{2-\beta}{2}
$$

then

$$
\frac{Q_{\beta}[v](z)}{z} \prec(1+z)^{1 / \beta} .
$$

Proof. Define a function $w(z), z \in U$ as follows:

$$
\left.\frac{z Q_{\beta}[v]^{\prime}(z)}{Q_{\beta}[v](z)}\right\}=(1+w(z))^{1 / \beta}, \quad z \in U
$$

where, $w(z)$ is analytic with $w(0)=0$. We need only to show that $|w(z)|<1$. From the definition of $w$, we have

$$
\frac{z Q_{\beta}[v]^{\prime}(z)}{Q_{\beta}[v](z)}=1-\beta \frac{z w^{\prime}(z)}{1+w(z)}
$$

Hence, we obtain

$$
\begin{aligned}
\operatorname{Re}\left\{\frac{z Q_{\beta}[v]^{\prime}(z)}{Q_{\beta}[v](z)}\right\} & =\operatorname{Re}\left\{1-\beta \frac{z w^{\prime}(z)}{1+w(z)}\right\} \\
& >\frac{2-\beta}{2}, \quad \beta \in(1,2) .
\end{aligned}
$$

In view of Lemma (1.2), there exists a complex number $z_{0} \in U$ such that $w\left(z_{0}\right)=e^{i \theta}$ and

$$
z_{0} w^{\prime}\left(z_{0}\right)=\kappa w\left(z_{0}\right)=\kappa e^{i \theta}, \kappa \geq 1
$$

Therefore, we arrive at

$$
\begin{aligned}
\operatorname{Re}\left\{\frac{z_{0} Q_{\beta}[v]^{\prime}\left(z_{0}\right)}{Q_{\beta}[v](z)}\right\} & =\operatorname{Re}\left\{1-\beta \frac{z w^{\prime}\left(z_{0}\right)}{1+w\left(z_{0}\right)}\right\} \\
& =\operatorname{Re}\left\{1-\beta \frac{\kappa w\left(z_{0}\right)}{1+w\left(z_{0}\right)}\right\} \\
& =1-\beta \operatorname{Re}\left\{\frac{\kappa e^{i \theta}}{1+e^{i \theta}}\right\} \\
& =\frac{2-\beta}{2},
\end{aligned}
$$

and this is a contradiction with the assumption of the theorem. Hence, there is no $z_{0} \in U$ with $\left|w\left(z_{0}\right)\right|=1$, which yields that $|w(z)|<1$. This completes the proof. 


\section{Applications}

We illustrate some applications of functions in the set $\mathcal{I}_{v}^{\beta}$ by using Theorem 2.1.

Example 3.1. Consider the function

$$
v(z)=\left(\frac{2}{7-\sigma}\right)(1-z)^{-\frac{5-\sigma}{2}-1}-\left(\frac{2}{7-\sigma}\right), \quad z \in U .
$$

Obviously, $v(0)=0$ and $v^{\prime}(0)=1$, where

$$
v^{\prime}(z)=(1-z)^{-\frac{5-\sigma}{2}-2}, \quad z \in U .
$$

The utility of the fact

yields

$$
z \rightarrow^{-} 1 \Rightarrow\left(\frac{z}{1-z}\right) \Rightarrow\left(-\frac{1}{2}\right)
$$

$$
\begin{aligned}
\operatorname{Re}\left(\frac{z v^{\prime \prime}(z)}{v^{\prime}(z)}+\frac{2}{\left(\frac{z v^{\prime}(z)}{v(z)}\right)}\right) & =-\left(\frac{5-\sigma}{2}-2\right) \operatorname{Re}\left(\frac{z}{1-z}\right)+\frac{2}{\left(\frac{7-\sigma}{2}\right) \operatorname{Re}\left(\frac{z}{1-z}\right)-\frac{2}{7-\sigma}}, \quad z \in U \\
& <\frac{5-\sigma}{4}-1+\frac{2}{7-\sigma}, \quad z \rightarrow^{-} 1 \\
& <\frac{(7-\sigma)(7-\sigma)-4(7-\sigma)+2(7-\sigma)}{4(7-\sigma)} \\
& =\frac{5-\sigma}{4} \\
& <\frac{5-\sigma}{2}, \quad \sigma \in(3,5) .
\end{aligned}
$$

Thus, in view of Theorem 2.1, $v \in \mathcal{I}_{v}^{\beta}$ and it is starlike in the open unit disk.

Example 3.2. Consider the function

$$
v(z)=\left(\frac{2}{5-\sigma}\right)(1-z)^{-\frac{3-\sigma}{2}-1}-\left(\frac{2}{5-\sigma}\right), \quad z \in U .
$$

Obviously, $\eta(0)=0$ and $\eta^{\prime}(0)=1$, where

$$
v^{\prime}(z)=(1-z)^{-\frac{3-\sigma}{2}-2}, \quad z \in U .
$$

Now, we attain

$$
\begin{aligned}
\operatorname{Re}\left(\frac{z v^{\prime \prime}(z)}{v^{\prime}(z)}+\frac{2}{\left(\frac{z v^{\prime}(z)}{v(z)}\right)}\right) & =-\left(\frac{3-\sigma}{2}-2\right) \operatorname{Re}\left(\frac{z}{1-z}\right)+\frac{2}{\left(\frac{5-\sigma}{2}\right) \operatorname{Re}\left(\frac{z}{1-z}\right)-\frac{2}{5-\sigma}}, z \in U \\
& =\frac{3-\sigma}{4}-1-\frac{8}{5-\sigma}, \quad z \rightarrow^{-} 1 \\
& <\frac{(3-\sigma)(5-\sigma)-4(5-\sigma)+2(5-\sigma)}{4(5-\sigma)} \\
& <\frac{(5-\sigma)(5-\sigma)-4(5-\sigma)+4(5-\sigma)}{4(5-\sigma)} \\
& =\frac{5-\sigma}{4} \\
& <\frac{5-\sigma}{2}, \quad \sigma \in(3,5) .
\end{aligned}
$$


Thus, in view of Theorem 2.1, $v \in \mathcal{I}_{v}^{\beta}$ and it is starlike in the open unit disk.

\section{Conclusion}

It is well known that the Tsallis entropy of order $\beta>0$ is concave and for $\beta<0$ is convex (see [6]). Here, we provided the complex entropy, that can satisfy the convexity and concavity for positive order (Theorem 2.1 and Theorem 2.3). An extended definition of Tsallis entropy in a complex domain is imposed based on the set of analytic functions. This definition is leaded to define a new class of analytic function, may call the class of geometric information of analytic functions. We introduced a sufficient condition, (1.1) of the existing. Moreover, under the same condition, the function was starlike (Theorem 2.1) and the integral of the first order was convex (Corollary 2.1). Some applications are shown the type of the normalized functions.

\section{RE F E R E N C E S}

1. R. Hernandez: Prescribing the preschwarzian in several complex variables, Annales Academiae Scientiarum Fennicae Mathematica, 36(2011), 331-340.

2. R.W. Ibrahim M.Z. Ahmad and H.F. Al-Janaby: Third-order differential subordination and superordination involving a fractional operator, Open Math., 13(2015) 706-728.

3. I. S. JACK: Functions starlike and convex of order $\alpha$, J.London Math.Soc. 3(1971), 469-474.

4. S.S. Miller and P.T. Mocanu: Differential Subordinations : Theory and Applications, Mrcel Dekker Inc., New York, 2000.

5. K. W. ONG , S.L. TAN and Y.E. TU: Integral operators and univalent functions, Tamkang Journal of Mathematics, 43(2012), 215-221.

6. C.Tsallis: Generalized entropy-based criterion for consistent testing, Physical Review E, 58(2) (1998), 1442.

Rabha W. Ibrahim

Faculty of Computer Science and Information Technology

Center of Cloud Computing

University Malaya, 50603, Malaysia

rabhaibrahim@yahoo.com 